\title{
Countering illegal UAV flights : passive DVB radar potentiality
}

\author{
Dominique Poullin \\ ONERA \\ Palaiseau, FRANCE \\ Dominique.poullin@onera.fr
}

\begin{abstract}
:
Since a few years, illegal UAV(Unmanned Aerial Vehicle) flights have been observed in different countries and under various environments[1]. Following these malicious fligths, most of the countries decided to counter such illegal activities asking their main industrial and laboratories to provide an already existing global solution against such UAV activities. However, it seems that up to now, none active system has been validated as the universal ideal solution.

This paper will analyse the main system requirements.

Then the potential of a passive DVB-T (Digital Video Broadcasting-Terrestrial) component will be illustrated using experimental data for both the detection, identification, location and alert functions:

- The expected benefits of such a passive DVB-T solution will be analysed by considering experimental results on multi-rotors $U A V$ as well as on fixed wings $U A V$.

- Furthermore, some limitations of such a passive countering UAV component/system will be outlined.

In summary, the passive DVB-T component offers very attractive capabilities for struggling against $U A V$ at ranges up to three kilometres and even more in terms of detection, identification, location and alert.The interest of passive component for struggling against UAV is quite new [2] and it has to be noticed that several papers were dedicated to such an application during the last Passive Coherent Location Meeting in Bonn Wachtberg in 2017[3][4][5].
\end{abstract}

\section{Introduction}

Considering the struggle against illegal UAV flights, Detection, Identification, Extraction, Location and Alert have to be addressed. After a short description of the exact meaning for all these functions in this paper, one important aspect (generally missing in the suggested solutions) will be outlined : the warning time.

A short overview of the main sensors currently suggested mainly for the survey component will be achieved and their main advantages and limitations outlined.

The main part of this paper will deal with a passive DVB-T (Digital Video Broadcasting Terrestrial) component that offers the opportunity of satisfying, at least for some scenarios/threats, simultaneously all the required functions with a short warning time without any requirement to another sensor. The potential of such a passive DVB-T approach will be illustrated using experimental data.

\section{UAV struggle requirements}

The UAV struggle requires the following functions :

- Detection : the UAV has to belong to the mobile objects detected and the surveillance has to be achieved in a wide sector. Ideally the observed sector has to be omnidirectional 
- Identification : in this paper identification refers only to a plot attribute/flag : 'UAV' or 'no interest'. The identification of the UAV class (quadcopter, hexacopter, ... fixed wing) may help for establishing a level of threat but is not compulsory for a first alert level.

- Extraction: the UAV plots (and attributes) have to be separated from the others and kept.

- Location : all the plots of interest (and especially the UAV) have to be located in cartesian coordinates according to the receiver site. A tracking will also help for predicting the UAV behaviour, but is not necessary for a first alert level.

- Alert : if a plot is identified as a UAV one and if its location is inside the forbidden perimeter: the alert has to be effective.

The identification process may imply two different mechanisms :

- The UAV plot maybe identified as a UAV directly by the plot signature or behaviour. In such a case the extraction will be simply based on the following consideration : if the plot is presenting the 'UAV behaviour' the plot is flagged as a suspicious one and extracted from the others.

- The UAV has no specific signature (or a too low signature level). However if some classes (cars, ...) may be identified, the extraction of plots of potential interest will be achieved by throwing away all the plots of non-interest. Consequently, if such an extraction is not ideal, it is helpful by limiting the number of identification requests to a dedicated identification component.

As mentionned previously, the first alert level could be simply achived by the combined direct identification of the UAV and its location inside a forbidden perimeter.

The main constraint for this UAV struggle is related to the short warning time:

- The UAV is a small object, so generally, at least for radar component, as far as the UAV may be detected, many other small targets such as birds and cars will be detected.

- The maximum detection range is generally quite small, typically a few kilometres

- The range requirement could be very small, between a few hundred of metres up to a few kilometres

- Considering a speed close to 20 metres per seconde the warning time should be not more than a few tenth of seconds.

The large number of potential detected targets for a surveillance system combined with the short warning time leads to a compulsory surveillance component with a high intrinsic capability of identification.

When considering a surveillance component detecting a typical situation with numerous targets detected in the domain of surveillance, if the surveillance component has to adress systematically a request to a dedicated identification component, the analysis of the whole domain, from a system (alert) point of view will take too many time for being efficient.

The real difficulty in the UAV struggle is not the detection but the first level of alert if your surveillance component has no identification capabilities and requires a systematic identification by another dedicated sensor.

Among the most common surveillance sensors it is possible to mention : 
- Radio-gonometers : good detection capabilities with identification ability but limited to remote controlled UAVs

- Acoustical component : detection capabilities with identification ability but restricted to short ranges (a few hundred of meters) configuration

- Battlefield radar : good detection capabilities but with limited identification ability (in their current version). Generally, such battlefield systems are scanning the azimuth space leading to short coherent integration time per scanned angle. Such a limited time analysis per scanned direction leads to a bad doppler resolution and consequently to troubles for detecting the blade modulation. Some new active systems try to overcome such a limitation by using wide aperture system [6].

- Passive DVB-T solution : good detection capabilities with interesting identification abilities. The rest of this paper will illustrate, with experimental data, such an antiUAV component/system capabilities.

\section{Short description of the DVB passive component}

DVB passive radar exploits the DVB-T illuminators of opportunity existing in its vicinity. Such broadcasters offer a quite omnidirectional illumination of all the low altitude receiver environment and this illumination is continuous all day and nigth long. The receiving DVB antennas are typically wide aperture antennas allowing a continuous surveillance of wide sectors without any time scheduling : all the 'directions' within the antenna elementary domain are simultaneously analysed (by signal processing) and all the detected plots may be located and identified (when possible) simultaneously.

Furthermore, as the DVB belongs to the low frequency radars, the Doppler analysis is intrinsically part of the passive signal processing. When analysing the effect of blades or propeller, such high rotating elements lead to a periodic received signal from the whole UAV target point of view and this period may be detected by this Doppler analysis.

Typically, the blade/propeller rotation is generally between 4000 revolutions per minute and 12000 revolutions per minute leading to a frequency period between $70 \mathrm{~Hz}$ and $200 \mathrm{~Hz}$. As the typical coherent integration time used for UAV detection is close to 0.5 secondes leading to a frequency resolution of $2 \mathrm{~Hz}$, such period of modulation may easily (if the level is sufficient) be detected by the classical Doppler analysis achieved in passive low frequency radar. This blade modulation frequency is independant from the radar carrier frequency and its detection is easier when long coherent integration time (related to high Doppler resolution) is available.

In summary, the classical passive DVB signal processing simply adapted, for UAV purpose, in terms of an increased coherent integration time up to 0.5 second or 1 second will simultaneously allow over all the sector of interest:

- UAV detection

- UAV identification by using the blade/propeller modulation detection (by simple Doppler/Fourier analysis) when this modulation presents a sufficient Signal to Noise Ratio (SNR). This level is mainly related to the blade material and length.

- UAV extraction (when direct identification is achieved)

- UAV location

- And, as a consequence of all these previous functions, UAV alert can be effective. 


\section{Situation : other typical targets detected}

As mentionned previously, a radar system able to detect small and medium UAV will also allow the detection of many other mobiles such as birds and terrestrial vehicles.

The next experimental results will illustrate a typical range domain occupied by cars and detected using DVB transmitter : the range 'tracks' with a positive evolution correspond to ground vehicles on one way of the road while the range tracks with negative evolution correspond to the vehicles on the other way.

For example in such a situation the typical number of detected target in our elementary antennas mainlobe (60 degrees in azimuth) and restricted to a few kilometers radius (from 3000 meters to 9000 metres for bistatic range) was equal to fourteen leading to an 'equivalent' omnidirectional situation with more than eighty targets for each Coherent Integration Time duration. Such a situation confirms the compulsory requirement of a survey component with identification ability in order to avoid a systematic identification for all the elementary plots as such a systematic identification will lead to a disaster from the alert warning time point of view.

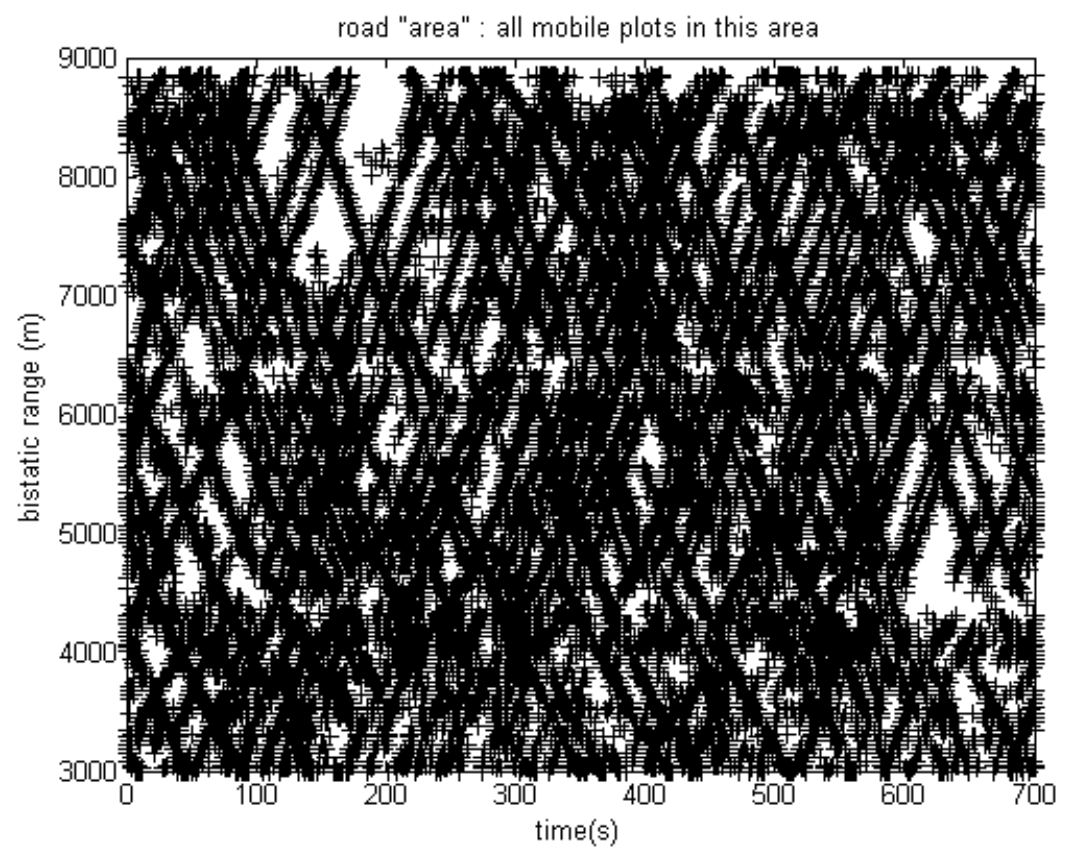

figure 1: example of typical situation of road detection

When a UAV is flying in such a complex situation, it is still possible to learn the ground vehicles behaviour and to not consider the detections with such ground vehicle parameters. This kind of 'unwanted target filter' could be implemented on the previous situation represented on figure 1 in order to avoid such a congested situation. However, such a filter is, of course, less efficient for countering (and selecting) UAV than a direct UAV identification as we'll illustrate in a next paragraph.

The figure 2 represents the output of such a basic filter and the average number of plots in the elementary domain considered (60 degrees in azimuth and 6000 meters for bistatic range) decreases from fourteen to 'half a target' (one plot every two scans). Some spurious are still remaining (here the filter is a basic one and, probably, it may be adapted more efficiently), however two targets (one close to time stamp 150 secondes and the other one to 550 secondes) are now clearly visibles. 


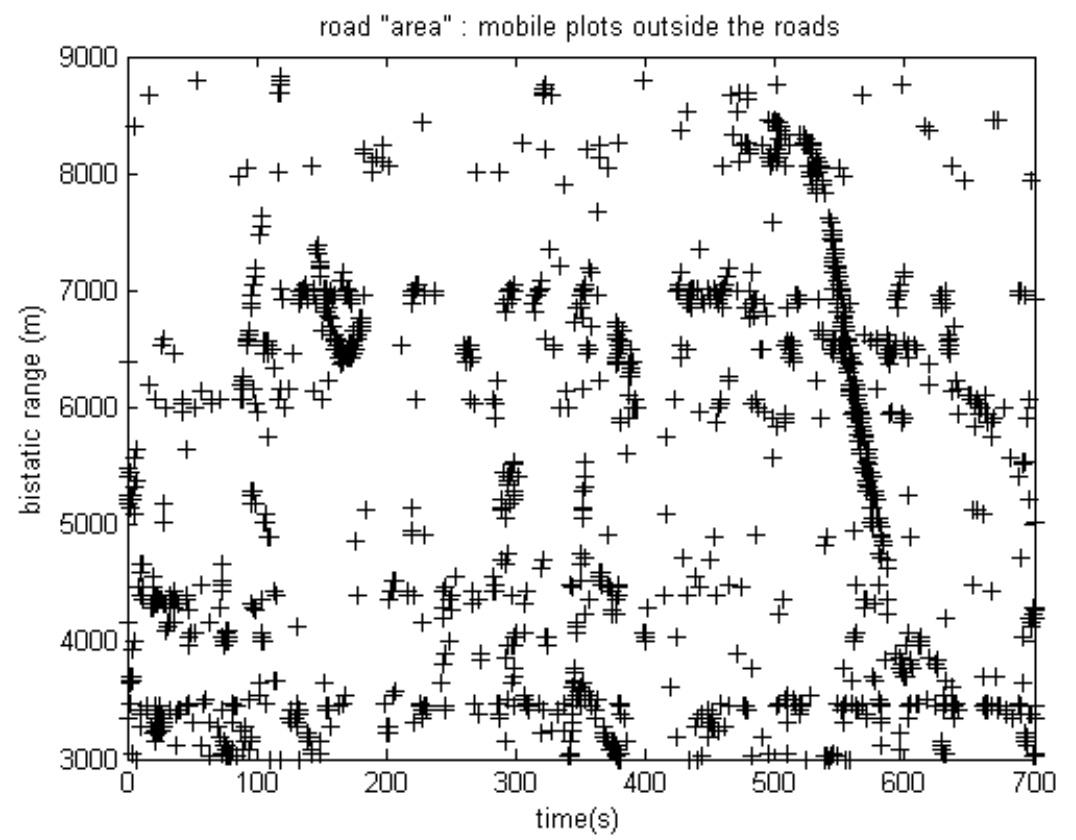

figure 2 : example of the range domain occupied by roads after 'car plots removal'

The next results on figure 3 is corresponding to a UAV trajectory crossed by a bird (buzzard). The clearly identified bird (by the UAV pilot) was the second linear track, and as this bird track is similar to the previous one, it is quite possible to guess that the previous linear track was also corresponding to another bird. When the bird track crosses the UAV one, these two mobile 'targets' were at 3 kilometers from the receiver.
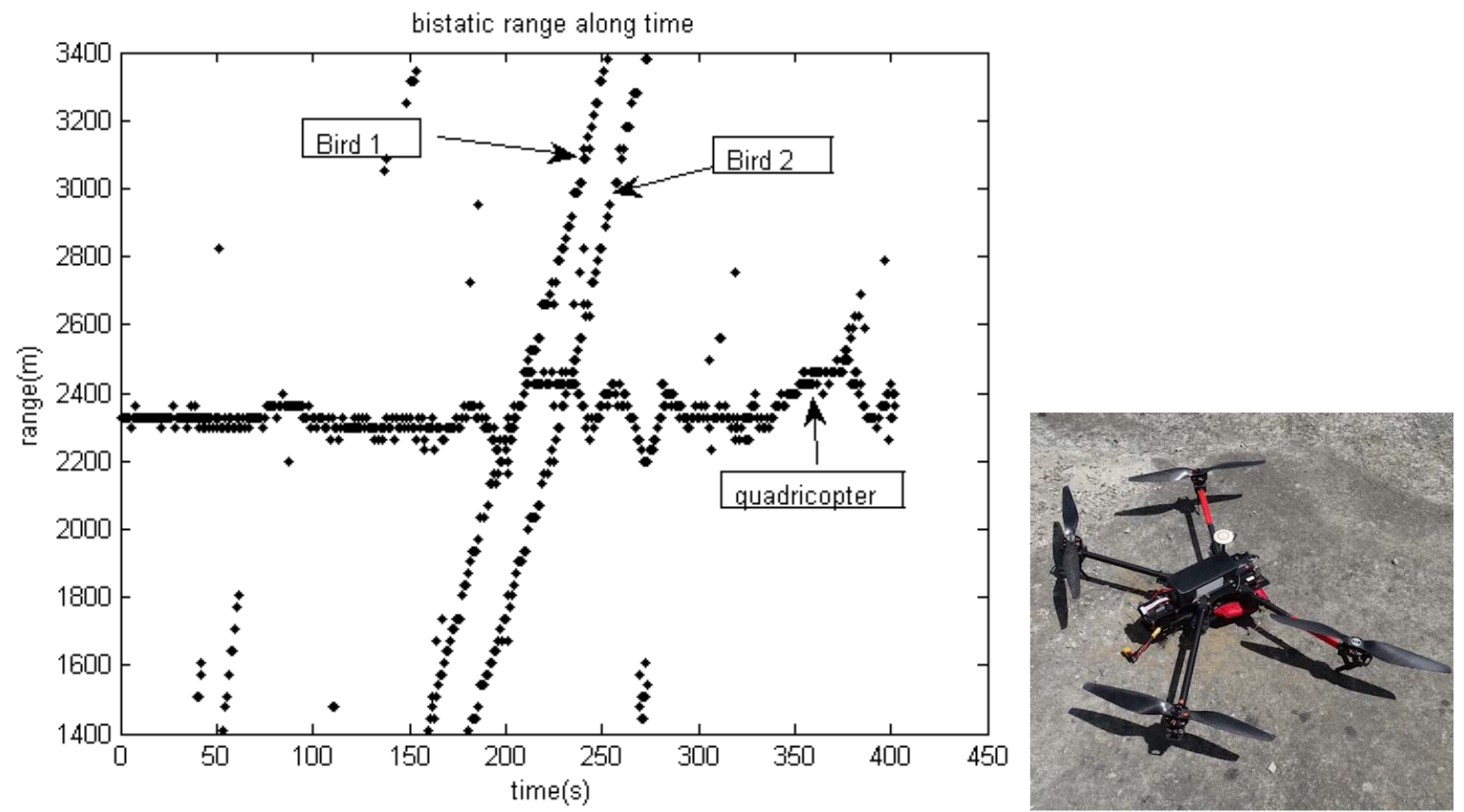

figure 3: example of a simultaneous detection of birds and quadcopter (see photo : UAV size is close to 50 centimetres)

\section{Passive DVB detection on multirotor UAV and fixed wings}

Now our intention is to illustrate the DVB ability to detect the UAV and its blade/propeller modulation. 
During this flight the quadcopter was stationnary leading to a zero Doppler evolution , however it was possible to detect the UAV by its blade modulation as illustrated on the figure 4. Obviously, the range and angle estimated on this blade modulation are similar to the range and angle UAV parameters allowing an 'indirect' UAV location.

The none detections at the beginning of the file are probably due to a too low altitude of the target: the UAV was hidden by trees until it reached an altitude allowing Line Of Sight between the UAV and the receiver (no ground truth was available during that flight).

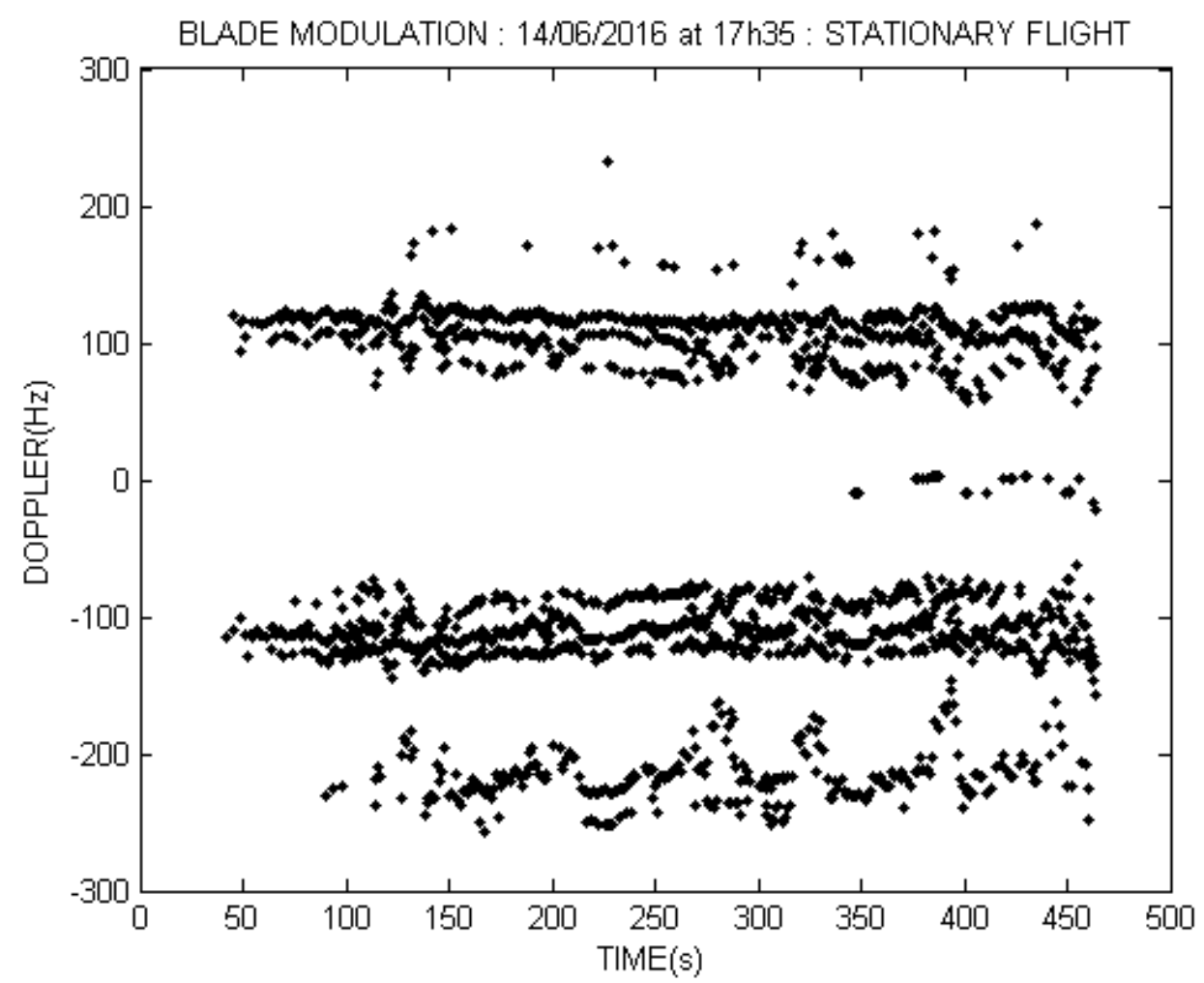

figure 4: example of quadrirotor blade modulation detected during a stationary flight at 3 kilometres

On figure 5 the UAV is following a trajectory based on circles around its launching position. On this result, we may guess the UAV detection (periodic range evolution especially in the second part of the record) for bistatic ranges close to 3500 meters. 


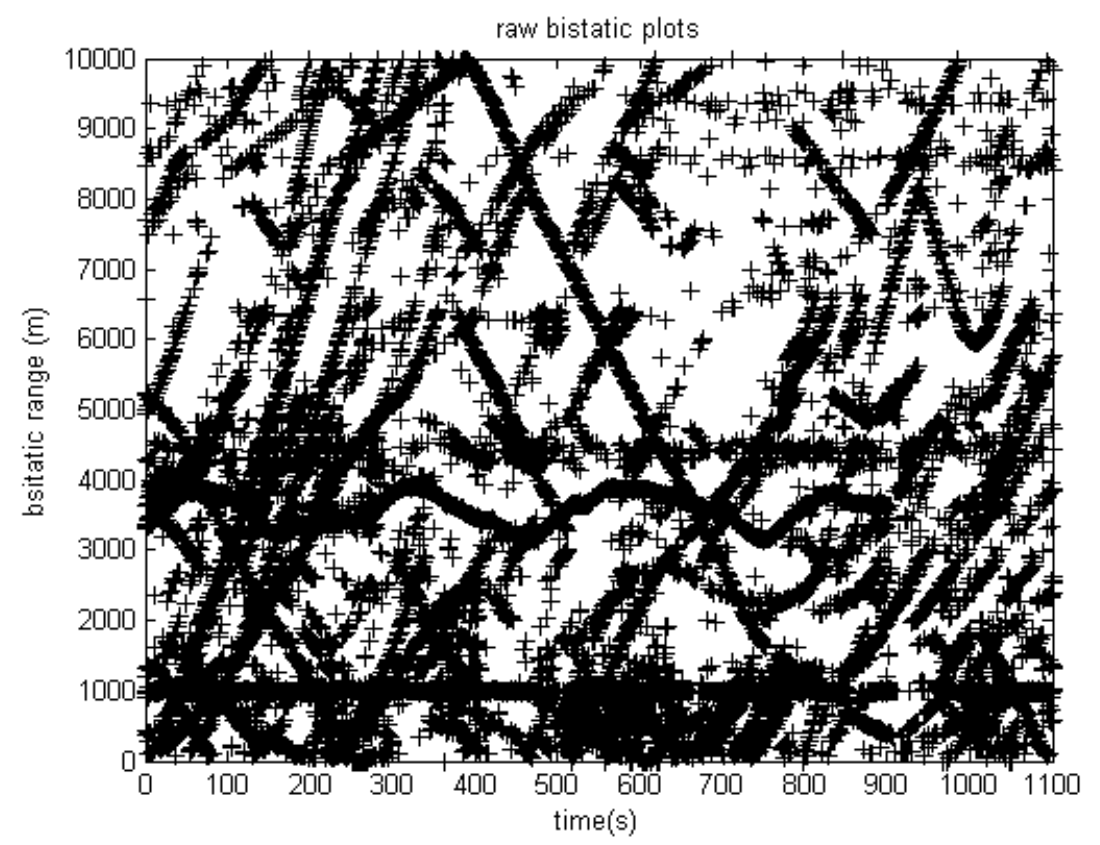

figure 5: example of a circular quadcopter flying in a typical quiet configuration

In such a situation, the UAV extraction is quite easy when using the flag parameter related to the blade modulation detection. When a plot is presenting detections at different 'Doppler' frequencies, the plot is stamped as a suspicious one as it corresponds to an object with highly rotating elements.

The figure 6 represents, among the previous detected plots (figure 5), the extracted suspicious ones. With such a 'blade' filter, the UAV is clearly extracted from all the other targets and its detection over the all file is verified. The small plots at the end of the file (close to time stamp 1000 secondes and 6000 meters of bistatic range) are not actual false alarms: they are corresponding to a small civilian plane and its propeller.

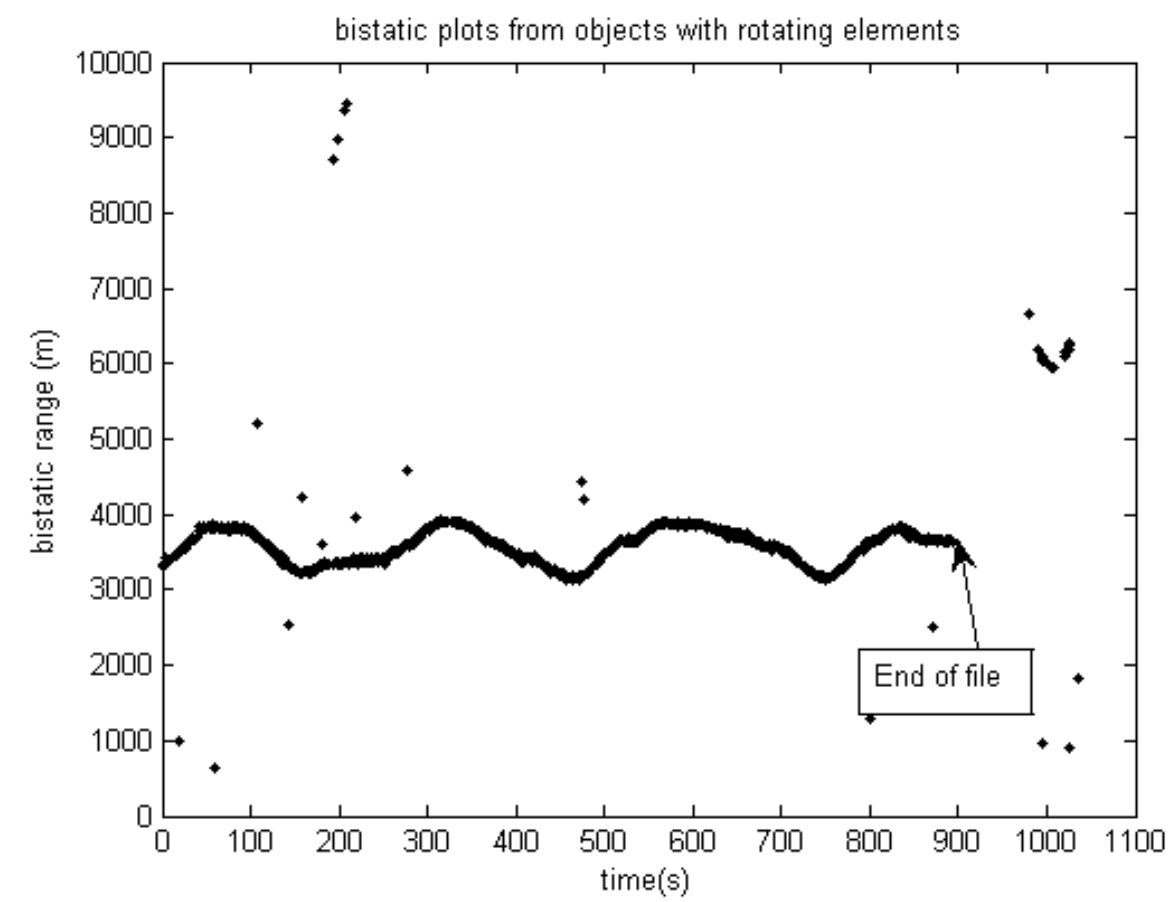

figure 6 : quadcopter extracted from the other targets by exploiting the blade modulation detection 
The figure 7 now illustrates the propeller detection of a fixed wing ( the 'Doppler' plots with a speed (absolute value) close to $50 \mathrm{~m} / \mathrm{s}$ ), the lower speeds are corresponding to the UAV cinematic itself. The propeller modulation is clearly different from the blade one especially due to the existence of different blade rotations for multi-rotors. However, in this paper the only element used is the 'blade/propeller' modulation existence for identifying the UAV, the intend was only to separate a UAV from the other mobile targets.

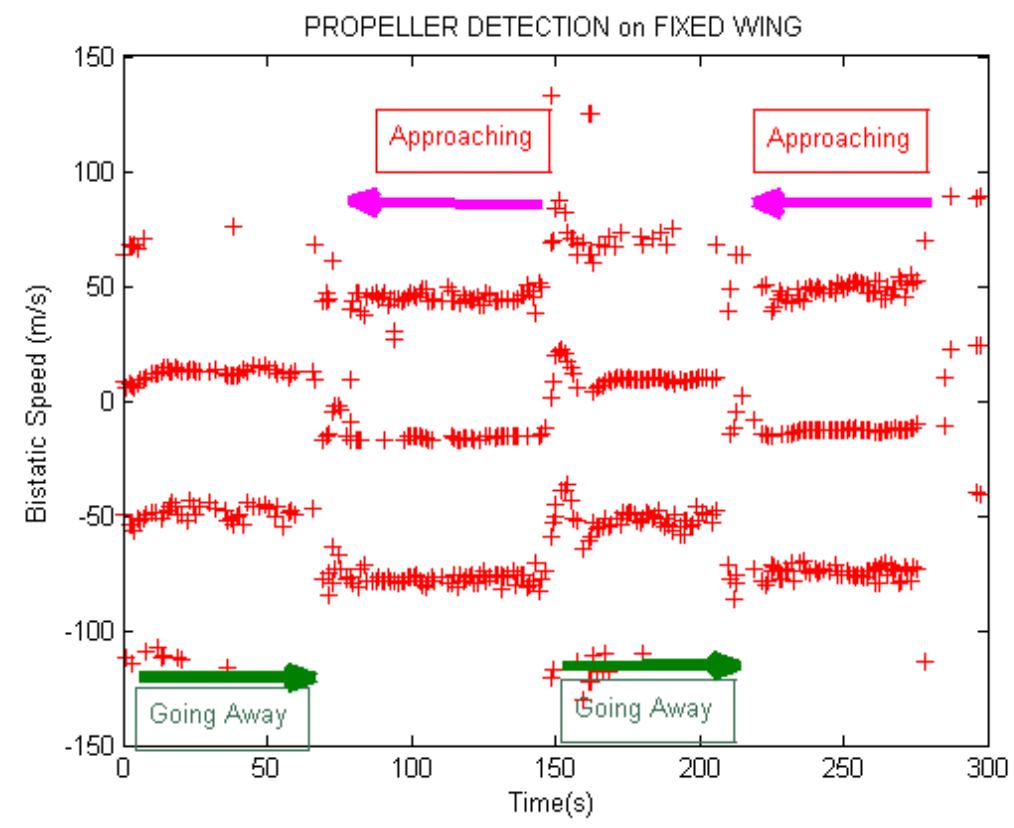

figure 7: example of propeller detection on fixed wing

In a similar way than for multirotors, such a propeller modulation allows the fixed wing extraction from the whole scene. The figure 8 and figure 9 illustrate this extraction in the measurement domain.

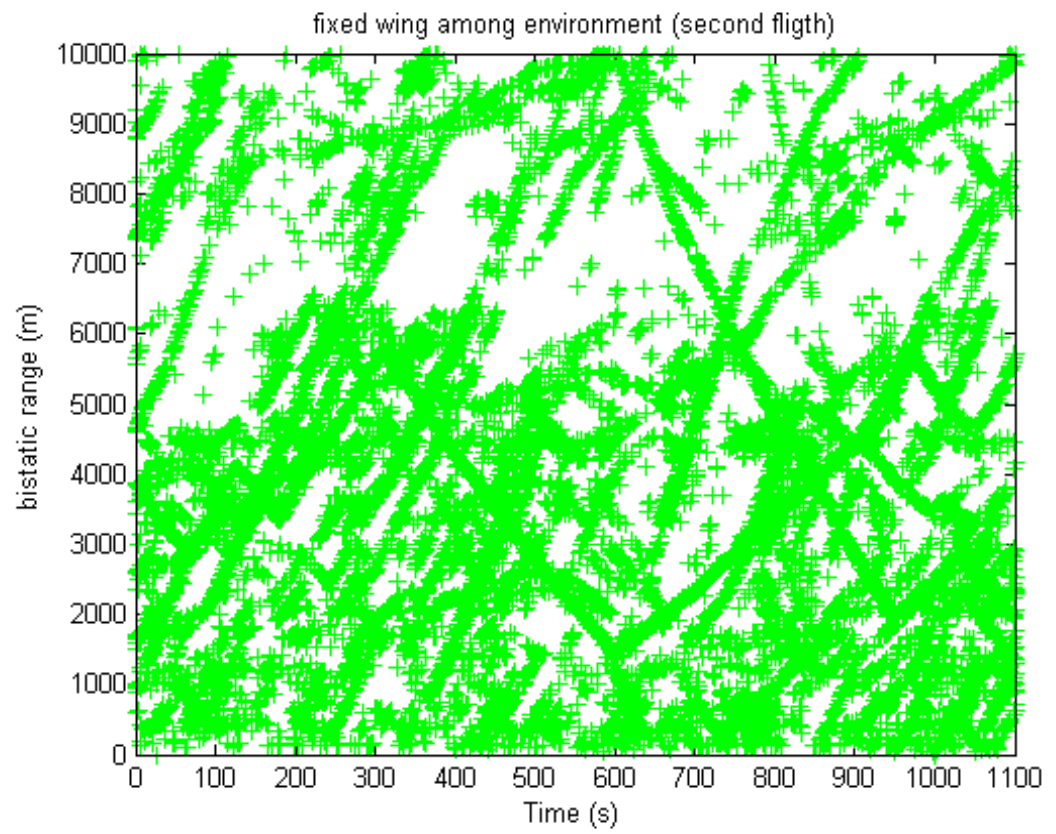

figure 8 : example of a fixed wing detected among several other mobile objects 


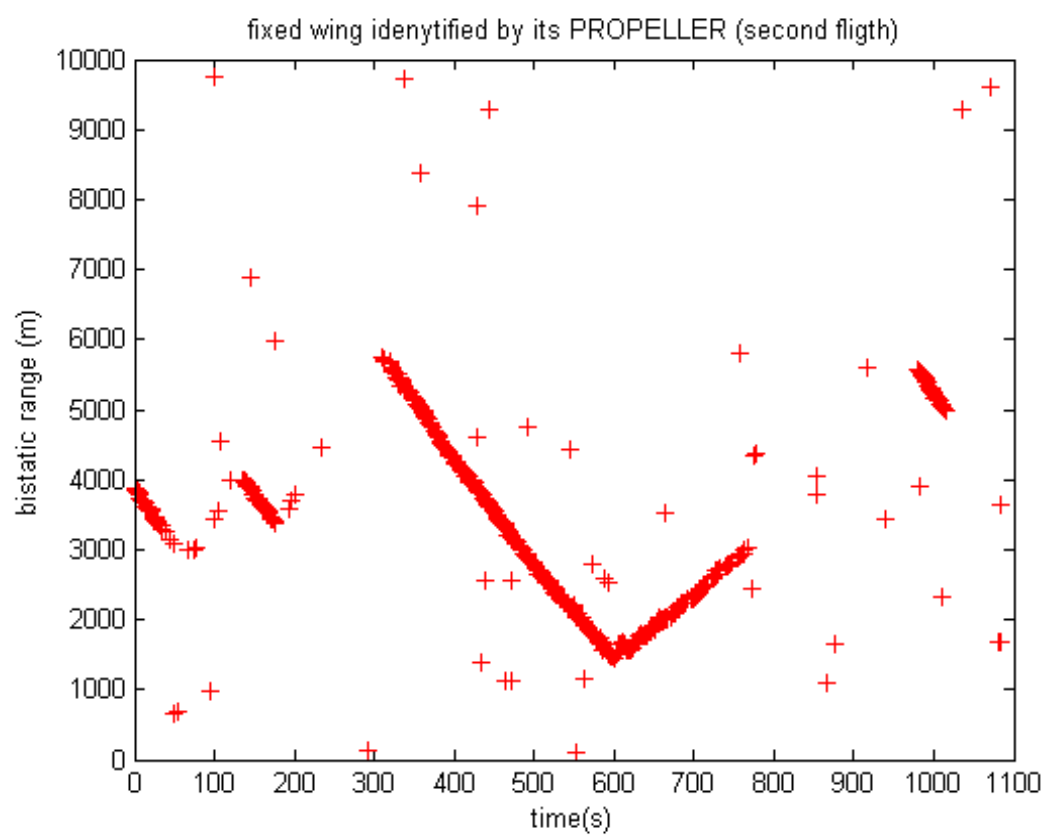

figure 9 : fixed wing detected and extracted from the scene by using its propeller modulation detection

\section{Location and alert}

The detected plots are then located by applying the geometrical transformation between the parameter measurement couple (bistatic range, azimuth angle) and the cartesian coordinates.

All the previous results have been achieved using a 8 receiving yagui antennas and the location of this fixed wing UAV, among the whole scene, is represented on the figure 10.

The green plots are corresponding to the whole scene while the red ones are related to plots with rotating elements.

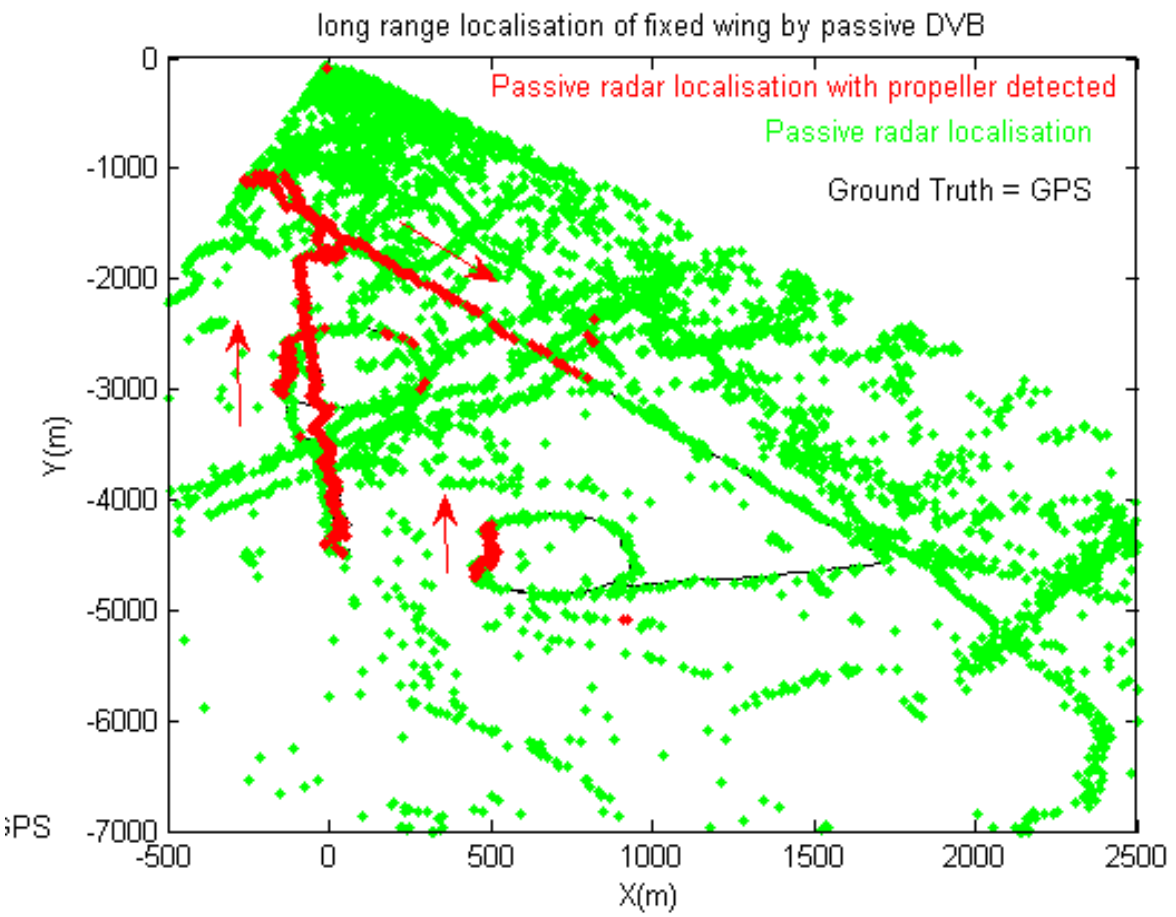

figure 10 : fixed wing UAV localized among the other mobile targets by bistatic passive DVB component 
It clearly appears that the propeller was correctly detected especially when the fixed wing was facing the receiver.

\section{7 conclusion}

The main difficulty in countering (if we except the neutralization aspect) UAV relies in the compulsory short warning time between the UAV appearance and the alert.

Most of the systems suggested based on a separated survey component and an identification one suffers from this compulsory requirement of a short warning time.

Passive DVB-T component/system offers the ability to cover simultaneously the different functions such as detection, identification, extraction, location and alert. This multi-functions component constitutes an efficient system to counter UAV at least up to quadcopter (medium size) and medium fixed wing UAV when the blades/propeller effects are detectable. Consequently this solution performances (in terms of system) is mainly dependent on the blades/propeller size and material. When such a direct UAV identification is no longer available, it is compulsory to implement some unwanted targets filters for avoiding a systematic identification (to another sensor) request for all the other targets.

Several experimental results have been presented in order to illustrate the good performances on passive DVB solution against UAV. The detection capabilities for both the quadcopter and the fixed wing UAV, were similar under the eight bistatic configurations that have been evaluated.

\section{References}

[1] https://www.chathamhouse.org/expert/comment/drones-are-increasing-security-issue-nuclearindustry

[2] D. Poullin UAV Detection and Localization Using Passive DVB-T Radar MFN and SFN MP-SET-231-18

[3] M. Malanowski, G. Krawczyk, M. Żywek, M. Szczepankiewicz, P. Samczyński. Drone detection experiment using DVB-T-based passive radar $6^{\text {th }}$ PCL meeting (2017)

[4] Tatiana Martelli, Fabiola Colone, Pierfrancesco Lombardo Detection and 3D localization of drones and ultralight aircrafts for a WiFi-based Passive Radar $6^{\text {th }}$ PCL meeting (2017)

[5] D Poullin, Passive DVB-T radar: UAV detection and identification $6^{\text {th }}$ PCL meeting (2017)

[6] http://www.aveillant.com/products/gamekeeper-16u/ 